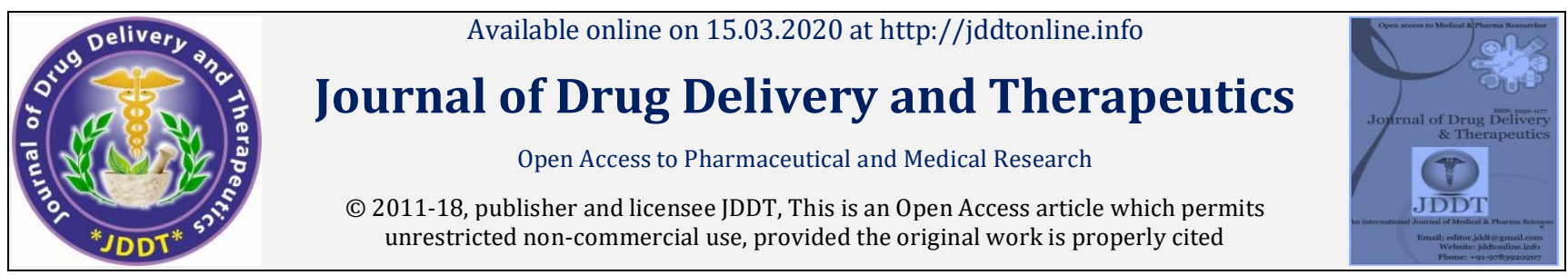

Open Access

Research Article

\title{
Drug Utilization Evaluation of Lorazepam in Alcohol Withdrawal Syndrome Patients: A Secondary Care Teaching Hospital Based Study
}

\author{
Divine Varghese*, Purnima Ashok, Shristi Nayak, Atul Aby Jacob, K. Vishnu Dev, Vijeesh Thollur \\ Department of Pharmacy Practice, K.L.E College of Pharmacy, Bengaluru-10, Karnataka, India
}

\begin{abstract}
Background: Drug utilization evaluation is an important parameter for the safe, effective and rational use of medications in medical care. Alcoholism results in abundant cases of alcohol withdrawal which can prove to be life-threatening if untreated, lorazepam is a drug of choice for Alcohol Withdrawal Syndrome and requires close monitoring as its over-dosing or under-dosing is common in case of withdrawal. Objective: ensures the safe and rational use of Lorazepam in the management of Alcohol Withdrawal Syndrome. Materials and Method: This prospective, observational study was conducted among alcoholic patients admitted in the KC General Hospital, Karnataka, India. Baseline data for Clinical Institute Withdrawal Assessment for Alcohol revised scale (CIWA-Ar) was collected on the day of admission and the response to lorazepam treatment was recorded using the same with respect to the baseline data every $12^{\text {th }}$ hourly after the initial administration until withdrawn. Results: Statistical analysis for 72 patients was done using ANOVA to calculate the overall progression of the syndrome with treatment. Among 72 patients $94.44 \%$ were male and $5.55 \%$ were female. The mean age of patients reported with AWS was found to be 44.90 years. A significant CIWA-Ar score reduction was observed with a p-value of $<0.0001$. Conclusion: Our study revealed a strong predominance of male patients with alcohol withdrawal syndrome, where maximum patients started consuming alcohol before the age of 20 years. After the administration of lorazepam, a significant CIWA-Ar score reduction was observed.
\end{abstract}

Keywords: Alcohol Withdrawal Syndrome, CIWA-Ar scale, Drug utilization evaluation, Lorazepam.

Article Info: Received 11 Jan 2020; Review Completed 19 Feb 2020; Accepted 28 Feb 2020; Available online 15 March 2020 Cite this article as:

Varghese D, Ashok P, Nayak S, Jacob AA, Dev KV, Thollur V, Drug Utilization Evaluation of Lorazepam in Alcohol Withdrawal Syndrome Patients: A Secondary Care Teaching Hospital Based Study, Journal of Drug Delivery and Therapeutics. 2020; 10(2):102-106 http://dx.doi.org/10.22270/jddt.v10i2.3991

*Address for Correspondence:

Divine Varghese, Department of Pharmacy Practice, K.L.E College of Pharmacy, Bengaluru-10, Karnataka, India

\section{INTRODUCTION}

Due to easy availability, liquor has become one of the most frequently used products which have had a universal effect.[1] According to the DSM-IV TR, Alcoholism, or alcohol use disorder (AUD) is the problematic pattern of alcohol use leading to clinically significant deterioration or psychological distress. Alcoholism results in an estimated 2.5 million death annually, representing $4 \%$ of all mortality.[2,3]

Chronic alcohol consumption leads to dependence and its cessation causes withdrawal syndrome, the severity of which is not mediated by the age, the extent of dependence, drinking patterns or prior number of detoxifications. ${ }^{[4]} \mathrm{A}$ significant number of AWS patients also have concurring liver disease or other medical problems. ${ }^{[5,6]}$

Alcohol in small amounts produce behavioral enticement and euphoria due to heightened glutamate binding to the
NMDA receptors. On the other hand, at high concentrations, it causes acute intoxication by the exponentiation of GABA effects.[7,8] During abstinence, NMDA function is augmented 20 and GABA transmission reduces resulting in withdrawal symptoms.[9] In AWS dopamine level also increases, which results in hallucinations and hyperarousal.[10]

The admissible therapy for treating alcohol withdrawal is with the use of Benzodiazepines [12] as Gradually tapering, Fixed-schedule or Symptom -triggered regimen accompanied by Non-pharmacological management by implementing early mobilization techniques, providing adequate intellectual support and environmental stimulation with light and noise control. [13,14] 
Table 1: Alcohol withdrawal syndrome- timeline and stages

\begin{tabular}{|l|l|}
\hline Time & Presentation \\
\hline $6-12$ hours (mild) & $\begin{array}{l}\text { Quiver, anxiety, GI upset, headache, insomnia, excessive sweating, palpitations, tachycardia, } \\
\text { hypertension. }\end{array}$ \\
\hline $12-24$ hours (Moderate) & Hallucinations (visual, auditory). \\
\hline $24-48$ hours & Withdrawal seizures \\
\hline $48-72$ hours (Delirium tremens) & Alcohol withdrawal delirium (delirium tremens) ${ }^{[11]}$ \\
\hline
\end{tabular}

Lorazepam is a Benzodiazepine indicated for the management of AWS[15] It is promptly absorbed orally and reaches maximum concentrations within 2 hours after administration. It is mainly metabolized in the liver by glucuronic acid conjugation to form inactive lorazepam glucuronide and has a t1/2 of 12 hours. ${ }^{16,17]}$

\section{Clinical Institute Withdrawal Assessment of Alcohol Scale-Revised (CIWA-Ar)}

It is a clinically effective, accurate, brief and simple scale to assess the alcohol withdrawal severity. This scale helps to monitor the response to treatment and ensures that optimal pharmacotherapy can be instituted in patients undergoing alcohol withdrawal.[18] Sixty-seven is the maximum possible score in the scale[19] and a score of $\geq 8$ but $\leq 15$ indicate a probable need for pharmacological treatment. If the score goes above fifteen there will be an increased risk of confusion and seizure[20] thus, it must be treated pharmacologically and vital signs monitoring is also important.[16] CIWA-Ar constitutes 10 withdrawal symptoms with a rating scale from 0 to 7 for nine of them and 0 to 4 for orientation.[21] Most of the symptoms in the scale can be rated through simple observation or by asking simple questions to assess orientation, headache, and visual/auditory or tactile disturbances. The symptoms specified within the CIWA-Ar scale are as follows:
1. Nausea/vomiting
2. Auditory disturbances
3. Paroxysmal sweats
4. Agitation
5. Tactile disturbances
6. Tremor
7. Visual disturbances
8. Headache
9. Orientation
10. Anxiety[22]

The severity of withdrawal can be assessed at the initial stage using the total CIWA-Ar score before initiation of pharmacotherapy. CIWA-Ar minimizes the under- and overdosing with BZD in the treatment of alcohol withdrawal. Most symptoms of alcohol withdrawal subside within four days. However, some symptoms, like anxiety and sleep disturbance may persist for longer.[23] This 10-item scale takes about one minute to complete by the assessor. Although it consumes very less time to administer, its relevance is still limited in research setting and is a factor limiting widespread clinical use.[24] CIWA-Ar is to be administered on the admission of the patient followed by scheduled hourly scoring until the score falls below 6 on four consecutive occasions.

\section{Drug utilization evaluation}

Drug Utilization Evaluation [DUE] or Medication Use Evaluation [MUE], according to WHO is the marketing, distribution, prescription and use of drugs in society with special prominence on the resulting medical social and economic consequences.[25] The purpose of DUE is to ensure the judicious, safe and effective use of drugs to improve patient health.[26] DUEs can be classified as qualitative and quantitative. Qualitative DUE studies are set out to determine the rational use of the drug. They require qualitative criteria such as duration of treatment, dosage for indications, drug of choice and fixed combinations.[27] Concurrent DUE studies are performed during the treatment course and the ongoing monitoring of drug therapy. $[25,26]$

\section{MATERIALS AND METHODS}

This prospective, observational study was conducted among 72 consecutive AWS patients both male and female admitted in the General Medicine department of KC General Hospital, Bengaluru, India from September 2018 to February 2019.

Patients between the age of 20 to 75 with a history of alcohol use and withdrawal syndrome prescribed with Lorazepam were enrolled in the study and written consent forms were obtained, after providing appropriate information. On obtaining Informed Consent Document, patient medical chart containing information such as demographics, past medical and medication, social and family history, medication chart, lab data, etc are recorded in the specifically prepared data collection forms. AWS patients prescribed with lorazepam were assessed for the efficacy and safety of the drug. Efficacy assessment of lorazepam was done using the CIWA-Ar scale score. Baseline data for CIWAAr was collected on the day of admission. The response to lorazepam treatment was recorded using the CIWA-Ar scale with respect to the baseline data every $12^{\text {th }}$ hourly after the initial administration until withdrawn. Safety assessment of lorazepam was done by examining for any drug-related errors, adverse events, interactions, etc. using Micromedex. Patients were counseled regarding their disease condition and alcohol abstinence and were provided with a Patient Information Leaflet after ascertaining their understanding.

Statistical analysis was done using repeated measure ANOVA followed by Takis multiple comparison test as the posttest to calculate the overall progression of the syndrome with treatment. 


\section{RESULTS}

\section{Gender distribution}

The prevalence of AWS was more among men (94\%) than women $(6 \%)$.

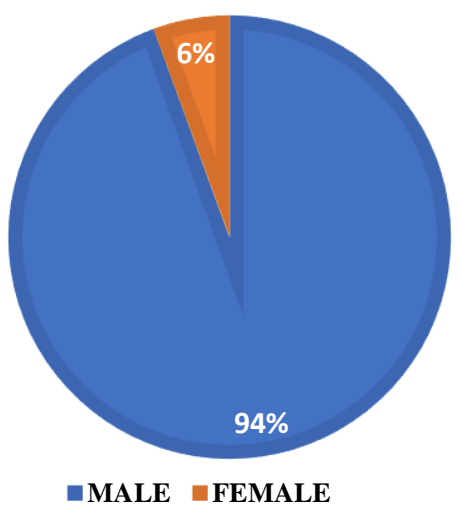

Figure 1: Frequency distribution of gender in the study population

\section{Age distribution}

The age distribution of patients with alcohol withdrawal cases in the age group of 20-45 constituted $42(58.33 \%)$ following by $30(41.66 \%)$ in the $46-75$ age group.

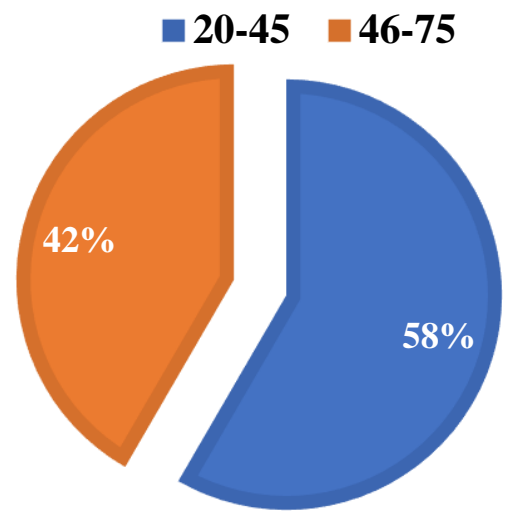

Figure 2: Age distribution

\section{The onset of alcohol consumption}

The age at which alcohol consumption began varied largely between $15-25$ years ( $40 \%$ ) to $>46 y$ years $(7 \%)$.

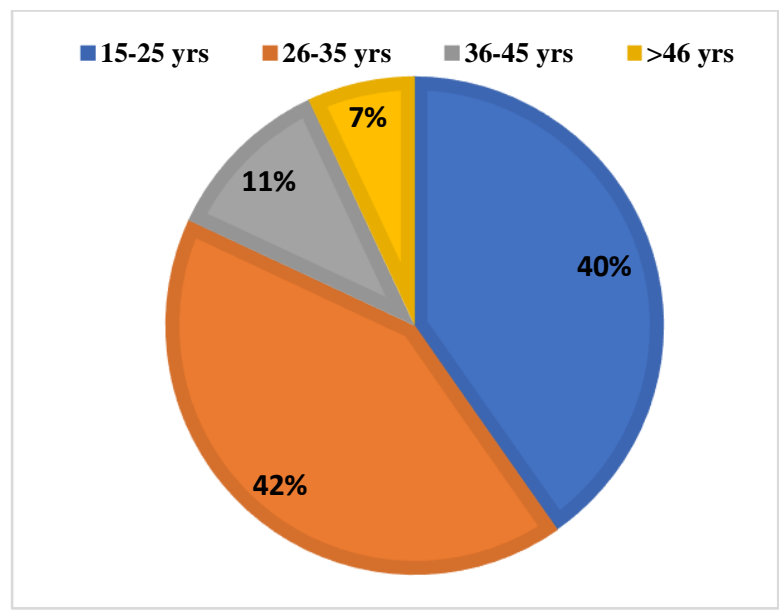

Figure 3: Frequency distribution for the onset of alcohol consumption

\section{Comorbid liver complications}

Out of 72 AWS patients, 45(62.5\%) presented with liver complications at various stages of alcoholic liver disease and $27(37.5 \%)$ patients with other accompanying diseases.

\section{Table 2: Comorbid liver conditions}

\begin{tabular}{|l|l|l|}
\hline & Patients & Percentage \\
\hline AWS with ALD & 45 & $63 \%$ \\
\hline AWS with other complications & 27 & $37 \%$ \\
\hline
\end{tabular}

\section{Reduction in CIWA-Ar score}

A significant CIWA-Ar score reduction was observed during the therapy. Results were obtained using Graph Pad Prism Version 5, Repeated measure ANOVA with

p-value of $<0.0001$.

\section{CIVA}
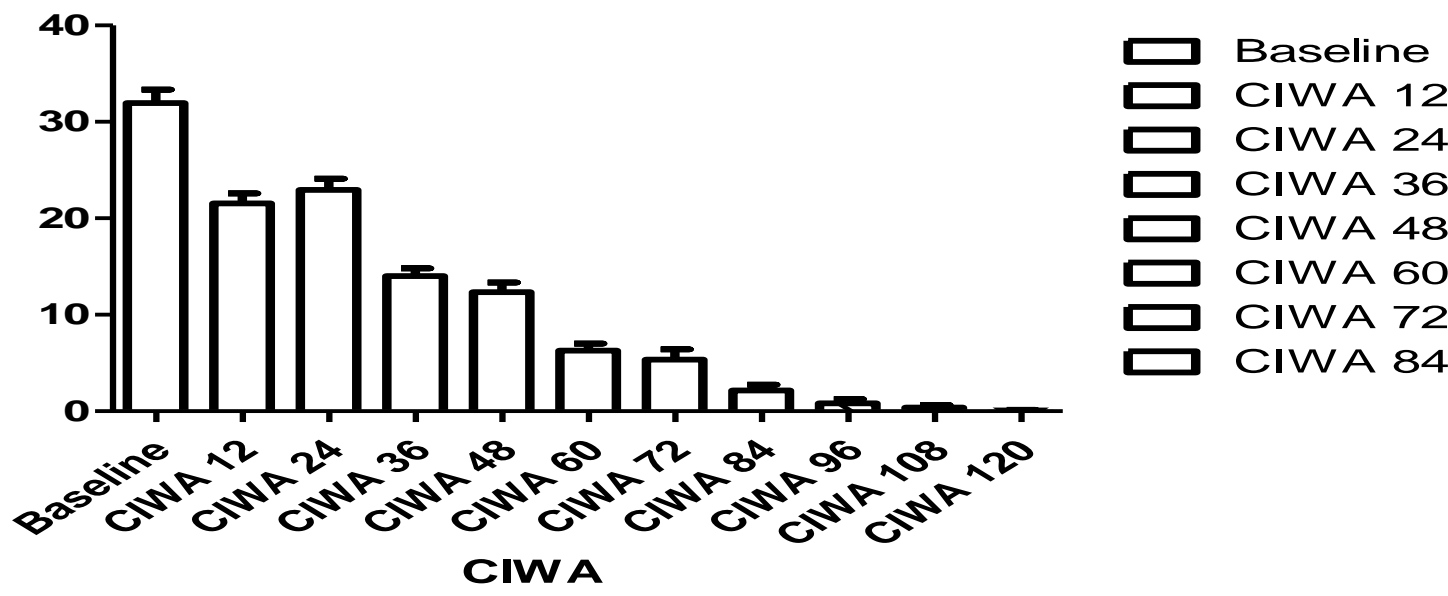

Figure 4: CIWA-Ar score after Lorazepam administration 


\section{Varghese et al}

\section{Time of symptom resolution}

Among 72 patients, on the administration of the CIWA-Ar scale, the score reduction corresponded to 10 symptoms of
Journal of Drug Delivery \& Therapeutics. 2020; 10(2):102-106

alcohol withdrawal. Among these symptoms, most patients recovered from nausea and tremors at the $60^{\text {th }}$ hour, At $48^{\text {th }}$ and $60^{\text {th-hour }}$ anxiety resolved while the remaining symptoms resolved at the $12^{\text {th }}$ hour.

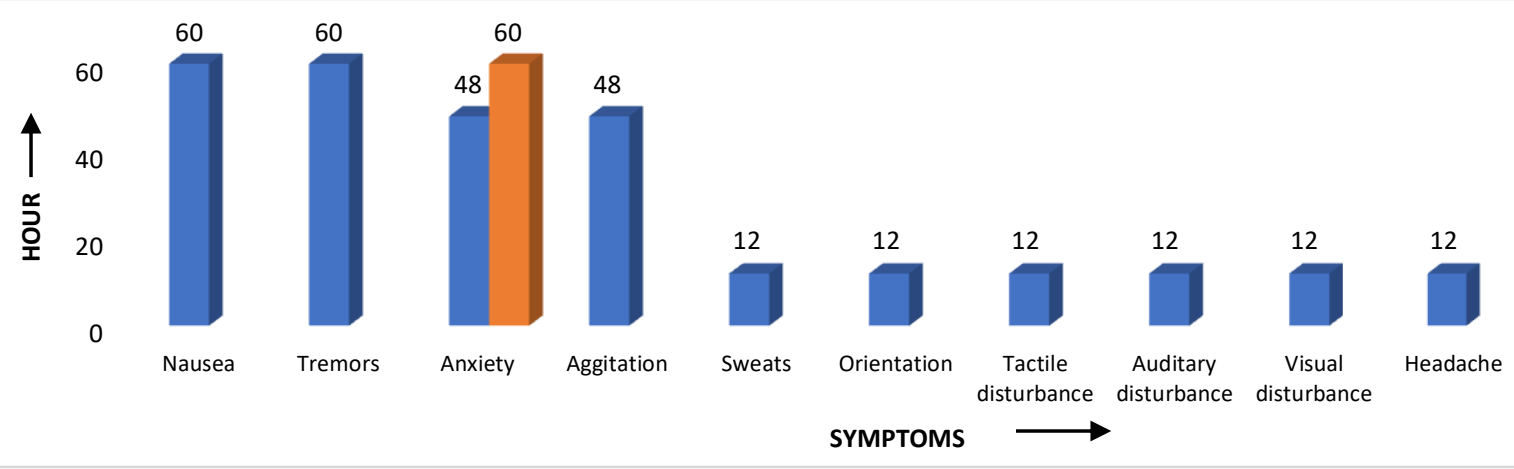

Figure 5: Frequency distribution of the time at which symptoms resolve.

\section{Recovery Distribution}

The maximum recovery was seen at the $60^{\text {th }}$ hour after Lorazepam administration with 43 patients followed by $48^{\text {th }}$ hour with 9 patients and $84^{\text {th }}$ hour with 8 patients and 96th hour with 4 patients, $72^{\text {nd }}$ hour with 3 patients and $36^{\text {th }}$ and $24^{\text {th }}$ hour with 2 patients each and $120^{\text {th }}$ hour with 1 patient.

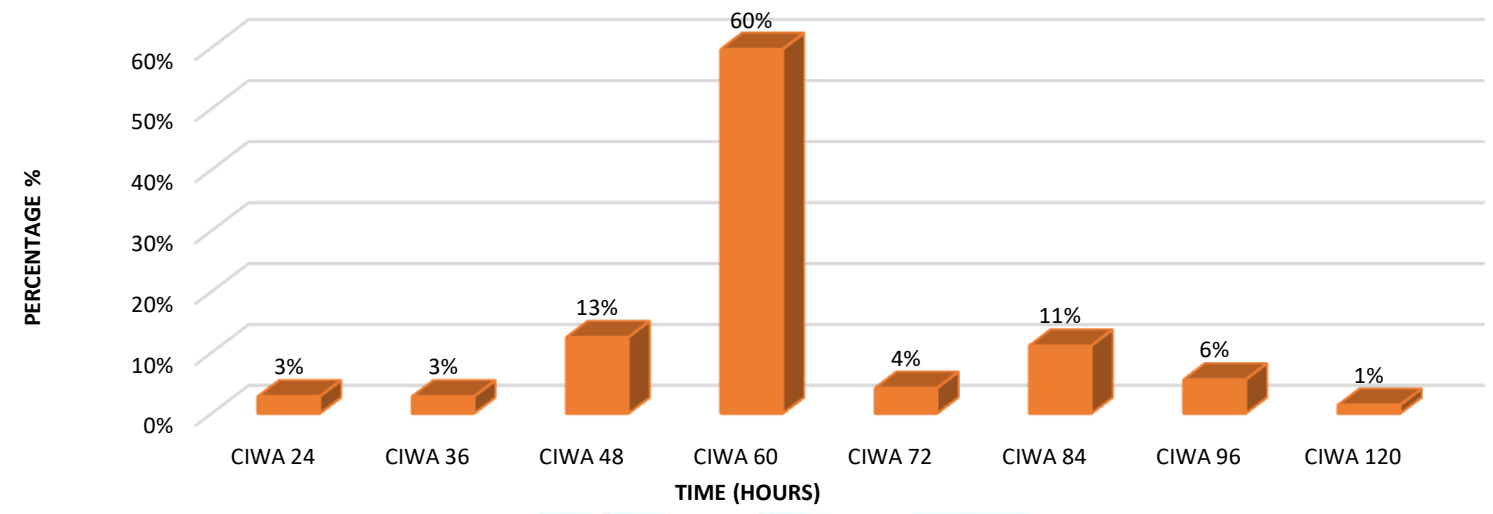

Figure 6: Recovery distribution

\section{DISCUSSION}

Alcohol withdrawal syndrome is a group of symptoms that occur on abruptly stopping after chronic alcohol consumption. Lorazepam is a short-acting, lipophilic benzodiazepine. It causes an increase of the GABA inhibitory effects by allosterically binding to the benzodiazepine receptors in its ligand-gated chloride channel of the central nervous system (CNS). This leads to cell membrane stabilization and hyperpolarization.

As per the WHO Global status report on alcohol and health, 2005 men surmount women in case of heavy drinking by four to one thus predominantly making them prone to alcohol-related problems than women which corresponds to result obtained in this study, where among 72 patients, 68 (94.44\%) were male and $4(5.55 \%)$ were females.[28]

As per the findings by Kolasani PB et al, the average age of alcoholic patients was 44.88 and the average age of patients enrolled in this study was found to be 44.90 years. This result also coincides with the observation by Wetterling $\mathrm{T}$ et $a l, 2000$ that the severity of AWS is age-independent. [3,5]

The study coordinated by Grant F.B et al 1997, summarized that a reduction of $8 \%$ alcohol abuse and $14 \%$ dependence was observed for every increase in age after onset, thus the chances of developing alcohol-related problems are more common among people who started at an early age which is contradicted in this study, where out of 72 patients, $29(40.28 \%)$ patients started consuming between age of 1525years, 30(41.67\%) started consuming between the age of 26-35 years, followed by $8(11.11 \%)$ patients between $36-45$ years and $5(6.94 \%)$ started above the age of 46 years. All these patients reported alcohol dependence and associated withdrawal.[29]

A majority of liver disease can be contributed to alcohol abuse. In our study, 45 (62.5\%) Alcohol withdrawal patients had accompanying liver complications at various stages of ALD and 27 (37.5\%) with other problems not related to liver functioning. As mentioned by Lorenzo Liggio et al, in their review alcoholism is the major factor in liver disease.[16]

As specified by Makdissi $\mathrm{R}$ et al and Asplund AC et al, the initial dose is 2-3 mg PO every 8-12 hours not exceeding $10 \mathrm{mg} /$ day. Maintenance dose is $2-6 \mathrm{mg} /$ day. All the patients in our study were prescribed with lorazepam (2mg) for withdrawal syndrome and evaluated every $12 \mathrm{hrs}$ for reduction in symptoms. [30,14]

Pursuant to Holbrook MA et al and Bayard M et al, we have adopted CIWA -Ar scale daily to check the efficacy of lorazepam therapy in arresting the withdrawal symptoms. A score on this scale lesser than 8 can be treated non- 
Varghese et al

pharmacologically while above 8 requires pharmacological therapy. [22,31]

Most symptoms of alcohol withdrawal subside within 4 days only a few may remain for longer. The severity of AWS can be assessed by CIWA-Ar scale and it checks the progress of treatment, wherein a decrease in the score after administration of lorazepam shows a positive response as recorded by Sullivan TJ et al and Gortney SJ et al, similarly, in our study, maximum patients recovered at the $60^{\text {th }}$ hour after the administration of Lorazepam with 43(59.72\%) patients. $9(12.5 \%)$ patients recovered at the $48^{\text {th }}$ hour $8(11.11 \%)$ at the $84^{\text {th }}$ hour, $4(5.55 \%)$ at the $96^{\text {th }}$ hour $3(4.16 \%)$ at the $72^{\text {nd }}$ hour and $2(2.77 \%)$ patients each at the $24^{\text {th }}$ and $36^{\text {th }}$ hour followed by $1(1.38 \%)$ at the $120^{\text {th }}$ hour. 34,48

On the administration of the CIWA-Ar scale, score reduction among the patients corresponded to 10 symptoms of alcohol withdrawal. Among these symptoms, most patients recovered from nausea and tremors at the $60^{\text {th }}$ hour. From anxiety, at both the $48^{\text {th }}$ and $60^{\text {th }}$ hour while the remaining symptoms resolved at the $12^{\text {th }}$ hour but this result contravenes with the study result done by S. Manikant et al in the utility of CIWA-A in AWS assessment. ${ }^{32}$

There was no moderate and severe adverse effect reported by the patients. Mild adverse effects like nausea, diarrhea, and sedation were reported by some patients and were selflimiting. No serious or irreversible side effects were observed throughout our study. Also, there was no significant drug interaction seen in the medication profile.

To identify the necessity of treatment, the severity of the baseline withdrawal manifestations had to be evaluated. The scale of 10 items scores the severity of vomiting, tremor, nausea, sweating, paroxysmal sweat, visual disturbances, anxiety, auditory disturbances, tactile disturbances, agitation, and headache. The dose is administered if the score is $>8^{39}$ and the scoring is repeated every $12^{\text {th }}$ hour until there is a reduction in the score. In our study among all 72 patients, significant CIWA-Ar scale score reduction was observed during the therapy. The results were obtained using Graph Pad Prism Version 5, Repeated measure ANOVA with a p-value of $<0.0001$.

\section{CONCLUSION}

In the treatment of AWS, lorazepam use within the study population was found to be safe and effective. A significant CIWA-Ar score reduction was observed during the therapy. No notable side effects and interactions were encountered in any of the patients.

\section{ACKNOWLEDGMENT}

We explicit our heartfelt gratitude to our Principal, H.O.D and Faculties of Pharmacy Practice Department, K.L.E College of Pharmacy for their invaluable support and guidance.

\section{CONFLICT OF INTEREST}

There was no conflict of interest among the authors.

\section{REFERENCES}

1. Fleming M, MihicJS, Harris RA. Ethanol. In: Hardman GJ, LimbirdEL. Goodman \& gilman's the pharmacological basis of therapeutics. 2001; 10:429-45.

2. Jesse S, Brathen G, Ferrara M, Keindl M, Ben-Menachem E, Tanasescu R et al. Alcohol withdrawal syndrome: mechanisms, manifestations and management. Acta Neurol Scand. 2017; 135(1):4-16.

3. Kolasani B, Sasidharan P, CM D, Jayabal P, Rajaseharan A. Prescribing pattern of drugs in patients with alcoholic liver
Journal of Drug Delivery \& Therapeutics. 2020; 10(2):102-106

disease in a tertiary care teaching hospital. Natl J Physiol, Pharm Pharmacol. 2017; 7(5):538-44.

4. Wetterling T, Driessen M, Kanitz DR, Junghanns K. The severity of Alcohol Withdrawal is not age dependent. Alcohol alcohol. 2001; 36(1):75-8.

5. Forrest E, Ahmed A, Benson G. The Management of Alcohol Withdrawal in Patients with advanced Liver Disease. Glasgow Royal Infirmary. 2013; 62(1):A83.

6. McKeon A, Frye AM, Delanty N. The Alcohol Withdrawal syndrome. J NuerolNeurosung Psychiatry. 2008; 79(8):854-62.

7. Hughes JR. Alcohol withdrawal seizures. Epilepsy Behav. 2009; 15(2):92-7.

8. Rogawski MA. Update on the neurobiology of alcohol withdrawal seizures. Epilepsy curr. 2005; 5(6):225-30.

9. Kovacs GL. Natriuretic peptides in alcohol withdrawal: central and peripheral mechanisms. Curr Med Chem. 2003; 10:2559-76.

10. S, Bharadwaj B. Clinical management of alcohol withdrawal: A systematic review. Ind Psychiatry J. 2013 Jul-Dec; 22(2):100-8.

11. Gortney SJ, Raub NJ, Patel P, Kokoska L, Hannawa M, Argyris A. Alcohol withdrawal syndrome in medical patients. Cleve Clin J Med. 2016 Jan; 83(1):67-79.

12. Mayo-smith MF, MD, Lee H.Beecher, Timothy L. Fischer, Gorelick, Jeanette L. Guillaume et al. Management of Withdrawal Delirium. N Eng J Med. 2004 July; 164(6):1405-12.

13. Sachdeva A, Choudhary M, Chandra M. Alcohol withdrawal syndrome: benzodiazepines and beyond. Journal of clinical and diagnostic research. 2015 Sep; 9(9):1-7.

14. Chad A. Asplund, Jacob W, Aaronson DO, Hadassah E. 3 regimens for alcohol withdrawal and detoxification. Fam Pract. 2004 Jul; 53(7):545-54.

15. Prasad, Manya; Krishnan, Pudukode R.; Sequeira, Reginald; AlRoomi, Khaldoon (2014-09-10). "Anticonvulsant therapy for status epilepticus". The Cochrane Database of Systematic Reviews. 2005 Oct;(4):1469-93

16. Leggio L, Lee M. Treatment of Alcohol Use Disorder in Patients with Alcoholic Liver Disease. Am J Med.2017; 130(2):124-34.

17. Rang PH, Dale MM, Ritter MJ, Moore KP. Anxiolytic and hypnotic drugs. Pharmacology. 2003; 5:515-23.

18. Sullivan J, Sykora K, Schneiderman J, Naranjo C, Sellers E. Assessment of Alcohol Withdrawal: the revised clinical institute withdrawal assessment for alcohol scale (CIWA-Ar). $\mathrm{Br}$ ) Addict.1989; 84(11):1353-57.

19. Vrdoljak D, Markovic B.B, Puljak L, Lalic I. D et al. Lifestyle intervention in general practice for physical activity, smoking, alcohol consumption and diet in elderly: A randomised controlled trial. Arch. Gerontol. Geriatr. 2014 Jan-Feb; 58(1):160-9.

20. Rajmohan V. An open label comparison of chlordiazepoxide and lorazepam in alcohol withdrawal. JEMDS. 2013 Jun; 2(25):460913.

21. Perry E. Inpatient Management of Acute Alcohol Withdrawal Syndrome. CNS Drugs 2014; 28(5):401-10.

22. Holbrook MA, Crowther R, Lottery A, Cheng C, King D. Diagnosis and management of acute alcohol withdrawal. CMAJ. 1999;160(5):675-80

23. Manikant S, Tripathi MB, Chavan SB. Utility of CIWA-A in alcohol withdrawal assessment. Indian J Psychiat. 1992; 34(4):347-5

24. Williams D, Lewis J, McBride A. A comparison of rating scales for alcohol withdrawal syndrome. Alcohol and alcoholism. 2001; 36(2):104-8.

25. Sachdeva P D. Drug utilization studies - scope and future pr. Ijpbr. 2010; 1(1):11-17.

26. Robert N. Managed care pharmacy practice, $2^{\text {nd }}$ ed. USA: Jones \& Bartlett Learning; 2008.

27. Sacristan AJ, Soto J. Drug utilization studies as tools in health economics. PharmacoEconomics. 1994; 5(4):299-312.

28. World Health Organization. Global status report on alcohol and health. Switzerland: World Health Organization; 2011

29. Grant FB, Dawson AD. Age at onset of alcohol use and its association with DSM-IV alcohol abuse and dependence. J Subst Abus Alcohol. 1997; 9:103-10.

30. Makdissi R, Stewart HS. Care for hospitalized patients with unhealthy alcohol use: a narrative review. Addict Sci Clin Pract. 2013; 8(11):1-10.

31. Bayard M, McIntyre J, Hill KR, Woodside J jr. Alcohol Withdrawal syndrome. Am Fam Physician. 2004 Mar; 69(6):1443-50. 